\title{
Prevalence and susceptibility to antibiotics from Campylobacter jejuni and Campylobacter coli isolated from chicken meat in southern Benin, West Africa
}

\author{
Sylvain Daton Kouglenou', Alidehou Jerrold Agbankpe ${ }^{2 *}$, Victorien Dougnon ${ }^{2}$, Armando Djiyou Djeuda $^{3}$, \\ Esther Deguenon², Marie Hidjo ${ }^{2}$, Lamine Baba-Moussa ${ }^{4}$ and Honore Bankole ${ }^{1,2}$
}

\begin{abstract}
Objective: Poultry is commonly considered to be the primary vehicle for Campylobacter infection in humans. The aim of this study is to assess the risk of Campylobacteriosis in chicken meat consumers in southern Benin by assessing the prevalence and resistance profile of Campylobacter coli and Campylobacter jejuni isolated from chicken thigh in Southern Benin.

Results: The contamination rate of Campylobacter in the samples was 32.8\%. From this percentage, 59.5\% were local chicken thighs and $40.5 \%$ of imported chicken thighs $(p=0.045)$. After molecular identification, on the 256 samples analyzed, the prevalence of C. jejuni was $23.4 \%$ and $7.8 \%$ for C. coli, with a concordance of 0.693 (Kappa coefficient of concordance) with the results from phenotypic identification. Seventy-two-point seven percent of Campylobacter strains were resistant to Ciprofloxacin, $71.4 \%$ were resistant to Ampicillin and Tetracycline. 55.8\% of the strains were multi-drug resistant.
\end{abstract}

Keywords: Campylobacter jejuni, Campylobacter coli, Chicken thighs, Antimicrobial resistance, Southern Benin

\section{Introduction}

Campylobacter, one of the food pathogens, has become one of the major causes of enteric infections in both developing and developed countries [1]. Incidence and prevalence of Campylobacteriosis has increased worldwide over the past decade, with approximately 500 million cases of gastroenteritis reported each year [2]. In Blantyre at Malawi, a 10-year study (1997-2007) found that C. jejuni and C. coli were detected in 21\% (415/1941 children) of hospitalized children with diarrhea by

\footnotetext{
${ }^{*}$ Correspondence: agbankpejerrold@yahoo.fr

${ }^{2}$ Research Unit in Applied Microbiology and Pharmacology of Natural Substances, Research Laboratory in Applied Biology, Polytechnic School of Abomey-Calavi, University of Abomey-Calavi, 01, P.O. Box 2009, Cotonou, Benin

Full list of author information is available at the end of the article
}

real-time PCR, with C. jejuni accounting for $85 \%$ of all campylobacteriosis cases [3]. Campylobacter transmission mainly occurs following exposure to farm animals with such infections, with subsequent passage through the food chain to retail food products $[4,5]$. Poultry are considered the main reservoir of infection and humans are most often infected by handling or consuming contaminated poultry meat [6].

Overuse or misuse of antimicrobials in the human population and in food animals has increased the number of antibiotic-resistant infections, including resistance to fluoroquinolones [7]. This resistance creates a real health problem, since the symptoms of campylobacteriosis are the same as those of gastrointestinal infections caused by other bacterial pathogens. Therefore, the empirical use of fluoroquinolones for the 
treatment of all gastrointestinal infections further promotes antibiotic resistance of this family [2]. In addition to the morbidity due to Campylobacteriosis and the risk of developing long-term sequelae, such as Guillian Barre syndrome (GBS), the development of antimicrobial resistance by Campylobacter strains constitutes an important concern [8].

A study in Benin reported contamination by Campylobacter spp. of imported poultry meat [9]. However, limited data exist on the health quality of local poultry meat about Campylobacter spp. In addition, it is important to know the resistance profile of these circulating Campylobacter strains in Benin. On this basis, we assessed the risk of Campylobacteriosis in chicken thighs consumers in southern Benin by determining the prevalence and susceptibility of Campylobacter strains isolated from samples of local and imported chicken meat commercialized in southern Benin.

\section{Main text \\ Methods \\ Study framework}

The sampling of chicken meat was carried out in the markets of the municipalities of Cotonou, Abomey-Calavi, Ouidah, Porto-Novo and Adjarra. All these markets are located in southern Benin. (Additional file 1: Figure S1). These communes were chosen because they belong to the three departments (Atlantique, Littoral and Oueme) which lead the pack when it comes to poultry farming in Benin [10].

\section{Sampling}

The sample size for this study was 256 . It was estimated using Schwartz's formula. The distribution of the different types of samples according to the markets and the municipalities is presented in Additional file 1: Table S1.

\section{Bacteriological isolation}

Samples were analyzed according to standard NF EN ISO 10272-1 modified [9, 11]. After enrichment and isolation on Karmali and Preston Campylobacter agars (at $42{ }^{\circ} \mathrm{C}$ in a microaerophilic atmosphere for $48 \mathrm{~h}$ ), a characteristic Campylobacter colonies was seeded on nutritive agar enriched with fresh sheep blood and incubated in a microaerophilic atmosphere at $37^{\circ} \mathrm{C}$ for $36 \mathrm{~h}$. Pure cultures obtained were stored in Müller Hinton $(\mathrm{MH})$ broth with glycerol $(30 \%)$ at $-37^{\circ} \mathrm{C}$ for additional analyzes.

The phenotypic identification of Campylobacter spp strains was carried out according to the methodology of Kougblenou et al. [11].

\section{Identification of isolated Campylobacter strains by Polymerase Chain Reaction (PCR)}

DNA of Campylobacter isolates were extracted using the Qiagen blue extraction kit. Molecular identification of isolates was carried out by PCR using 16SrRNA (816 bp) primer specific to all species Campylobacter: C412F 5'-GGATGACACTTTTCGGAGC-3' ${ }^{\prime}$ and C1228R 5'-CATTGTAGCACGTGTGTC-3' [12, 13]. Then, the isolates were identified as $C$. jejuni and $C$. coli using specific primers. C. jejuni (mepA (413 bp)): CJmapAN3F 5'-TGGTGGTTTTGAAGCAAAGA-3 'and CImapAN3R 5'-GCTTGGTGCGGATTGTAAA-3' [11, 13]; C. coli (ceuE (330 bp)): CCceuEN3F 5'-AAG CGTTGCAAAACTTTATGG-3'and CCceuEN3R' 5-CCTTGTGCGCGTTCTTTATT-3' $[12,14]$. The PCR products was run on gel electrophoresis. For each PCR reaction, two positive controls were carried out using the reference strains C. jejuni ATCC 29428 and C. coli ATCC 33559.

\section{Antibiotic susceptibility testing of Campylobacter isolates}

The determination of the sensitivity to antibiotics was carried out on all strains isolated, according to the method of diffusion on disc [15]. The choice of the 6 antibiotics tested (Additional file 1: Table S2) is justified by the fact that, the drugs of choice used in the clinical therapy of campylobacteriosis are macrolides, quinolones, fluoroquinolones and tetracyclines [15-18].

\section{Statistical analysis}

Data were analyzed with statistical software $R$ version 3.6.1. The difference was significant when $p<0.05$. In addition, a $95 \%$ confidence interval $(95 \% \mathrm{CI})$ was also determined for antibiotic resistance rates.

\section{Results}

\section{Results of bacteriological culture of samples}

$32.8 \%$ of all chicken thigh samples analyzed tested positive for Campylobacter culture. Of the 84 positive samples, 50 (59.5\%) were local chicken thighs and 34 (40.5\%) were imported chicken thighs, representing respectively $39.1 \%$ of the samples from local chicken thighs and $26.6 \%$ of imported chicken thigh samples (Additional file 1: Table S3). This difference in frequency of positivity is statistically significant $(p=0.045)$ between the samples of local and imported chicken thighs.

\section{Campylobacter isolation}

The PCR results showed that out of all the 80 phenotypically identified Campylobacter strains (C. jejuni and C. coli), the identity of 77 were confirmed. Of the 77 
confirmed strains, 45 were isolated from local chicken thighs and 32 were from imported chicken thighs. Of the 60 strains of $C$. jejuni obtained after phenotypic identification 55 were confirmed $C$. jejuni, 2 strains were identified $C$. coli and three strains which are neither C. jejuni nor C. coli. That is to say $91.7 \%$ of identity match of the strains of $C$. jejuni. As for the 20 phenotypically identified $C$. coli strains, 14 were confirmed $C$. coli and 6 C. jejuni by PCR, i.e. $70 \%$ of identity match of the C. coli strains (Table 1 and Additional file 1: Figure S2). Analysis of these results showed that there is a concordance between the results of the phenotypic identification and those of the molecular identification with the coefficient of kappa which is equal to 0.693 .

\section{Antibiotic resistance of identified Campylobacter strains}

The antibiotic susceptibility test was carried out on the 77 strains whose species level identities were confirmed by PCR. $72.7 \%$ of these strains were resistant to ciprofloxacin, $71.4 \%$ were resistant to ampicillin and tetracycline respectively, $19.5 \%$ of strains showed resistance to amoxicillin + clavulanic acid, $11.7 \%$ showed resistance to erythromycin and $7.8 \%$ showed resistance to gentamicin (Table 2). Analysis of the results showed that the difference in resistance percentages between the strains of $C$. jejuni and $C$. coli with ampicillin $(\mathrm{p}=0.001)$ and tetracycline $(\mathrm{p}=0.030)$ is statistically significant. The strains of $C$. jejuni and $C$. coli did not show strong resistance to erythromycin $(C$. jejuni (4.9\%); C. coli (37.5\%); 95\% CI $=2.0-79.6)$, but the difference in their resistance percentages was statistically significant $(\mathrm{p}=0.001)$.

The distribution of antibiotic resistance among Campylobacter strains has shown that the number of antibiotic resistant strains differs from one municipality to another, depending on the antibiotic disc (Additional file 1: Figure S3). This discrepancy in the level of antibiotic resistance is statistically significant between the five municipalities with regard to amoxicillin + clavulanic acid $(p=0.015)$, ampicillin $(\mathrm{p}=0.001)$ and gentamicin $(\mathrm{p}=0.016)$.

Table 1 Comparison of identification of Campylobacter species by PCR with that obtained phenotypically

\begin{tabular}{|c|c|c|c|c|c|c|c|c|}
\hline \multirow[t]{4}{*}{ Municipalities } & \multicolumn{8}{|c|}{ Type of samples n (\%) } \\
\hline & \multicolumn{4}{|c|}{ Local chicken thighs } & \multicolumn{4}{|c|}{ Imported chicken thighs } \\
\hline & \multicolumn{2}{|c|}{ Phenotypic identification } & \multicolumn{2}{|c|}{ Identification by PCR } & \multicolumn{2}{|c|}{ Phenotypic identification } & \multicolumn{2}{|c|}{ Identification by PCR } \\
\hline & C. jejuni & C. coli & C. jejuni & C. coli & C. jejuni & C. coli & C. jejuni & C. coli \\
\hline Abomey-Calavi & $8(24.2)$ & $4(28.6)$ & $8(23.5)$ & $4(36.4)$ & $8(29.6)$ & 0 & $7(26.0)$ & 0 \\
\hline Adjarra & $3(9.1)$ & $3(21.4)$ & $5(14.7)$ & $1(9.1)$ & $5(18.5)$ & $1(16.7)$ & $5(18.5)$ & $1(20.0)$ \\
\hline Cotonou & $8(24.2)$ & $2(14.3)$ & $6(17.6)$ & $2(18.2)$ & $4(14.8)$ & $2(33.3)$ & $4(14.8)$ & $2(40.0)$ \\
\hline Ouidah & $5(15.2)$ & $1(7.1)$ & $6(17.6)$ & 0 & $4(14.8)$ & $2(33.3)$ & $4(14.8)$ & $2(40.0)$ \\
\hline Porto-Novo & $9(27.3)$ & $4(28.6)$ & $9(26.5)$ & $4(36.4)$ & $6(22.2)$ & $1(16.7)$ & $7(26.0)$ & 0 \\
\hline Total & 33 & 14 & 34 & 11 & 27 & 6 & 27 & 5 \\
\hline
\end{tabular}

Table 2 Susceptibility of $C$. jejuni and C. coli strains identified to the different antibiotics used

\begin{tabular}{|c|c|c|c|c|c|c|c|c|}
\hline \multirow[t]{2}{*}{ Families of antibiotics used } & \multirow[t]{2}{*}{ Antibiotics used } & \multirow{2}{*}{$\begin{array}{l}\text { All strains } \\
\text { identified } \\
n=77(\%)\end{array}$} & \multicolumn{3}{|c|}{ Type of samples } & \multicolumn{3}{|c|}{ Campylobacter species identified } \\
\hline & & & $\begin{array}{l}\text { Local } \\
\text { chicken } \\
\text { thighs } \\
\mathrm{n}=45(\%)\end{array}$ & $\begin{array}{l}\text { Imported } \\
\text { chicken } \\
\text { thighs } \\
\mathrm{n}=32(\%)\end{array}$ & $95 \% \mathrm{Cl}^{\mathrm{b}}$ & $\begin{array}{l}\text { C. jejuni } \\
\mathrm{n}=61(\%)\end{array}$ & $\begin{array}{l}\text { C. coli } \\
\mathrm{n}=16(\%)\end{array}$ & $95 \% \mathrm{Cl}^{\mathrm{b}}$ \\
\hline \multirow[t]{2}{*}{$\beta$-Lactams } & AMC & $15(19.5)$ & $10(22.2)$ & $5(15.6)$ & $0.4-6.4$ & $12(19.7)$ & $3(18.8)$ & $0.1-4.3$ \\
\hline & AMP & $55(71.4)$ & $31(68.9)$ & $24(75.0)$ & $0.2-2.3$ & $49^{\mathrm{a}}(80.3)$ & $6^{\mathrm{a}}(37.5)$ & $0.0-0.6$ \\
\hline Fluoroquinolones & CIP & $56(72.7)$ & $35(77.8)$ & $21(65.6)$ & $0.6-5.7$ & $42(68.9)$ & $14(87.5)$ & $0.6-31.1$ \\
\hline Macrolides & E & $9(11.7)$ & $6(13.3)$ & $3(9.4)$ & $0.3-10.0$ & $3^{\mathrm{a}}(4.9)$ & $6^{\mathrm{a}}(37.5)$ & $2.0-79.6$ \\
\hline Tetracycline & $\mathrm{TE}$ & $55(71.4)$ & $33(73.3)$ & $22(68.8)$ & $0.4-3.8$ & $40^{\mathrm{a}}(65.6)$ & $15^{\mathrm{a}}(93.8)$ & $1.0-346.5$ \\
\hline Aminoglycosides & GM & $6(7.8)$ & $3(6.7)$ & $3(9.4)$ & $0.1-5.5$ & $4(6.6)$ & $2(12.5)$ & $0.2-15.8$ \\
\hline
\end{tabular}

AMC Amoxicillin + Clavulanic Acid, AMP Ampicillin, CIP Ciprofloxacin; Erythromycin, TE Tetracycline, GM Gentamicin

a Statistically significant difference between the two proportions

b $95 \%$ Confidence interval 
With regard to the multidrug resistance of the isolated strains, 43 (55.8\%) strains of Campylobacter were resistant to at least 3 antibiotics belonging to three different families. 26 (60.5\%) of these strains were resistant to 3 antibiotics, 13 (30.2\%) were resistant to 4 antibiotics, $3(7 \%)$ to 5 antibiotics and one (2.3\%) to 6 antibiotics (Table 3).

\section{Discussion}

Out of the 256 chicken thigh samples analyzed, 84 samples were positive for Campylobacter spp culture, representing a $32.8 \%$ contamination rate. In the five municipalities involved in this study, these rates are 40.6\% in Adjarra, 32.8\% in Abomey-Calavi and PortoNovo, $37.5 \%$ in Ouidah and $26.6 \%$ in Cotonou (Additional file 1: Table S3). Although these contamination rates are different from one municipality to another, there is no statistically significant difference between the level of contamination in these municipalities ( $p>0.05)$. This means that consumers of chicken meat from any of the municipalities involved in this study are almost similarly exposed to the risk of Campylobacter foodborne infections. Moreover, the obtained results have shown a statistically significant lower contamination rate of imported chicken thighs (26.6\%) compared to the local ones $(39.1 \%)(p=0.045)$. These results are in agreement with those found by [9] in Benin, where the prevalence of contamination of poultry meat imported into Benin was $20 \%$. The high contamination rate of local chicken meat compared to that of imported meat, shows that there is a lack of hygiene during the production of local chicken meat. Thus, Benin has not yet reached the level of hygiene recommended by the World Health Organization and the joint committee of the Food and Agriculture Organization of the United Nations, when handling products meat [19].

In the present study, the prevalence is $23.8 \%$ for $C$. jejuni and $6.3 \%$ for $C$. coli in chicken meat in southern Benin. This prevalence is low, but higher than those obtained in the spring ( $11 \%$ for $C$. jejuni, $0 \%$ for C. coli), in summer ( $11 \%$ for C. jejuni, $0 \%$ for C. coli) and in winter $(2.5 \%$ for $C$ jejuni, $1 \%$ for $C$. coli) in broilers in Tunisia [8]. The strains of $C$. jejuni (55.7\%) and C. coli (68.8\%) were found mostly in the samples of local chicken thighs. These results are in agreement with those found by [20], where the prevalence of C. jejuni (46.1\%) and C. coli $(32.8 \%)$ in poultry meat in Korea is very high than that obtained in poultry meat imported into the country.

Several studies have shown resistance of Campylobacter strains to fluoroquinolones, tetracyclines and macrolides [2, 17, 21-25]. The susceptibility of Campylobacter strains isolated in the present study showed $72.7 \%$ resistance to fluoroquinolones (Ciprofloxacin), $71.4 \%$ resistance to tetracyclines (Tetracycline) and $\beta$-lactams (Ampicillin). The high level of resistance of the strains to these three antibiotics was observed in strains isolated both from local and imported chicken thighs. High resistance rate of Campylobacter strains to these three antibiotics has also been observed in Algeria on Campylobacter strains isolated from turkeys [24]. In northern

Table 3 Multi-resistance profiles of the strains identified according to the type of sample and the Campylobacter species

\begin{tabular}{|c|c|c|c|c|c|c|}
\hline \multirow[t]{2}{*}{$\begin{array}{l}\text { Number of antibiotics } \\
\text { from different families }\end{array}$} & \multirow[t]{2}{*}{ Multi-resistance profiles } & \multirow{2}{*}{$\begin{array}{l}\text { All strains } \\
\text { identified } \\
n=77(\%)\end{array}$} & \multicolumn{2}{|c|}{ Type of samples } & \multicolumn{2}{|c|}{$\begin{array}{l}\text { Campylobacter } \\
\text { species identified }\end{array}$} \\
\hline & & & $\begin{array}{l}\text { Local chicken } \\
\text { thighs } \\
n=45(\%)\end{array}$ & $\begin{array}{l}\text { Imported chicken } \\
\text { thighs } \\
n=32(\%)\end{array}$ & $\begin{array}{l}\text { C. jejuni } \\
\mathrm{n}=61\end{array}$ & $\begin{array}{l}\text { C. coli } \\
\mathrm{n}=16\end{array}$ \\
\hline \multirow[t]{5}{*}{3} & $A M P-C I P-T E$ & $21(48.8)$ & $11(40.7)$ & $10(62.5)$ & $21(60.0)$ & 0 \\
\hline & AMC-AMP-TE & $2(4.7)$ & $1(3.7)$ & $1(6.3)$ & $2(5.7)$ & 0 \\
\hline & CIP-E-TE & $1(2.3)$ & $1(3.7)$ & 0 & 0 & $1(12.5)$ \\
\hline & AMC-CIP-TE & $1(2.3)$ & $1(3.7)$ & 0 & 0 & $1(12.5)$ \\
\hline & $A M C-A M P-C I P$ & $1(2.3)$ & $1(3.7)$ & 0 & $1(2.9)$ & 0 \\
\hline \multirow[t]{4}{*}{4} & AMC-AMP-CIP-TE & $5(11.6)$ & $4(14.8)$ & $1(6.3)$ & $5(14.3)$ & 0 \\
\hline & $A M P-C I P-E-T E$ & $6(14.0)$ & $5(18.5)$ & $1(6.3)$ & $2(5.7)$ & $4(50.0)$ \\
\hline & AMP-CIP-TE-GM & $1(2.3)$ & 0 & $1(6.3)$ & $1(2.9)$ & 0 \\
\hline & $A M C-A M P-C I P-G M$ & $1(2.3)$ & 0 & $1(6.3)$ & $1(2.9)$ & 0 \\
\hline \multirow[t]{2}{*}{5} & AMC-AMP-CIP-TE-GM & $2(2.6)$ & $2(7.4)$ & 0 & $1(2.9)$ & $1(12.5)$ \\
\hline & $A M C-A M P-C I P-E-T E$ & $1(2.3)$ & 0 & $1(6.3)$ & $1(2.9)$ & $0(12.5)$ \\
\hline 6 & AMC-AMP-CIP-E-TE-GM & $1(2.3)$ & $1(3.7)$ & 0 & 0 & $1(12.5)$ \\
\hline Total & & $43(55.8)$ & $27(60.0)$ & $16(50.0)$ & $35(57.4)$ & $8(50.0)$ \\
\hline
\end{tabular}


Tunisia, Campylobacter strains isolated from chicken meat samples showed strong resistance to ampicillin (61.4\%), ciprofloxacin (99.2\%) and tetracycline (100\%) [8]. These results can be explained by the common and sometimes uncontrolled use of the same antibiotics in poultry farms to fight against bacterial infections on farms. Indeed, a clear association between the use of fluoroquinolones and tetracyclines in poultry production and the high resistance rate of Campylobacter isolated from poultry has been shown by several studies [26-28]. The strains of $C$. jejuni are more resistant to ampicillin than those of C. coli. While resistance to tetracycline and erythromycin is more observed in strains of $C$. coli than in strains of $C$. jejuni. These results are in agreement with those found by [17] and [4].

More than half (55.8\%) of the isolated Campylobacter strains were multidrug-resistant with at least three antibiotics belonging to three different families and twelve determined multidrug resistance profiles. These data show how often the emergence of multidrug resistance in bacterial strains is increasing in Benin. Such alarming percentages of multidrug resistance of Campylobacter strains were also observed in other countries, $96.6 \%$ in Algeria [24], 90\% in South Africa [29] and 69\% in Poland [17]. The AMP-CIP-TE resistance profile was most observed in the strains of C. jejuni (52.5\%). This pattern has been observed with similar frequency in Korea with strains of $C$. jejuni isolated from poultry meat samples [20]. Molecular characterization of resistance genes is imperative for a better understanding of gene transmission and the mechanisms behind these resistances.

\section{Conclusion}

Results from this study show that there is a real risk of Campylobacter poisoning among consumers of chicken meat in southern Benin. In addition, these isolated Campylobacter strains are multidrug-resistant, which poses a problem in selecting Campylobacter strains in chicken farms, where antibiotics are used in an anarchic manner.

\section{Limitations}

Absence of characterization of resistance genes, and sequencing of Campylobacter genome constitutes the limit of this study.

\section{Supplementary information}

Supplementary information accompanies this paper at https://doi. org/10.1186/s13104-020-05150-x.

Additional file 1: Figure S1. Map of southern Benin showing the study area and sampling sites; Table S1. Distribution of different types of samples; Table S2. List of antibiotics tested and their respective loads;
Table S3. Distribution of culture results according to the nature of the samples, markets and municipalities where the samples were taken; Figure S2. Photo of agarose gel electrophoresis of amplicons of some Campylobacter strains isolated from chicken thighs; Figure S3. Frequency of resistance of Campylobacter strains as a function of the sampling area.

\section{Abbreviations}

GBS: Guillian Barre Syndrome; AMP: Ampicillin; GM: Gentamicin; E: Erythromycin; CIP: Ciprofloxacin; TE: Tetracycline; AMC: Amoxicillin + clavulanic acid; Cl: Confidence interval; PCR: Polymerase Chain Reaction; MH: Müller Hinton.

\section{Acknowledgements}

The authors thank all the trainees of the Food Microbiology Laboratory of the Ministry of Health of Benin for their involvement during the sampling. We are also grateful to Dr. Razaki Osse and Mr. Wilfrid Sewade for their technical support during the molecular identification of Campylobacter isolates.

\section{Authors' contributions}

SDK, JA, VD, ADD, ED, MH, LB-M and HB wrote the protocol. SDK, JA, VD, ED and $\mathrm{MH}$ processed the samples. JA and ADD did the statistical analyses. SDK, $J A$ and $A D D$ wrote the draft of the manuscript. JA, VD, LB-M and HB reviewed the manuscript. All authors read and approved the final manuscript.

\section{Funding}

No external funding was obtained to carry out this study. It was carried out with own funding from the authors and co-authors.

\section{Availability of data and materials}

All data generated or analysed during this study is included in this published article and Additional file.

\section{Ethics approval and consent to participate}

The present study was not performed on humans or animals, so it does not require the ethical approval. The samples analyzed as part of this study consist of chicken meat that we bought in the markets of southern Benin.

\section{Consent to publish}

The current manuscript contains no individual person's data. Therefore consent to publish is not applicable.

\section{Competing interests}

The authors declare that they have no competing interests.

\section{Author details}

${ }^{1}$ Laboratory of Food Microbiology, Ministry of Health, 01, P.O. Box 418, Cotonou, Benin. ${ }^{2}$ Research Unit in Applied Microbiology and Pharmacology of Natural Substances, Research Laboratory in Applied Biology, Polytechnic School of Abomey-Calavi, University of Abomey-Calavi, 01, P.O. Box 2009, Cotonou, Benin. ${ }^{3}$ Laboratory for Public Health Research Biotechnologies, Biotechnology Center, University of Yaounde I, P.O. Box 812 Yaounde, Cameroon. ${ }^{4}$ Laboratory of Biology and Molecular Typing in Microbiology, Faculty of Science and Technology, University of Abomey-Calavi, 05, P.O. Box 1604, Cotonou, Benin.

Received: 27 March 2020 Accepted: 22 June 2020

Published online: 26 June 2020

\section{References}

1. Rawat N, Maansi DK, Upadhyay AK. Virulence typing and antibiotic susceptibility profiling of thermophilic Campylobacters isolated from poultry, animal, and human species. Vet World. 2018;11(12):1698-705.

2. Marotta F, Garofolo G, Di-Marcantonio L, Di-Serafino G, Neri D, et al. Antimicrobial resistance genotypes and phenotypes of Campylobacter jejuni isolated in Italy from humans, birds from wild and urban habitats, and poultry. PLOS ONE. 2019;14(10):e0223804. https://doi.org/10.1371/journ al.pone.0223804.

3. Mason J, Iturriza-Gomara M, O'Brien SJ, Ngwira BM, Dove W, Maiden MC, Cunliffe NA. Campylobacter infection in children in Malawi is common 
and is frequently associated with enteric virus coinfections. PLOS ONE. 2013;8:e59663. https://doi.org/10.1371/journal.pone.0059663.

4. Lim S-K, Moon D-C, Chae MH, Kim HJ, Nam H-M, Kim S-R, Jang G-C, Lee K, Jung S-C, Lee HS. Macrolide resistance mechanisms and virulence factors in erythromycin-resistant Campylobacter species isolated from chicken and swine feces and carcasses. The Journal of Veterinary Medical Science. 2016;78(12):1791-5.

5. Mughini-Gras L, Smid JH, Wagenaar JA, de Boer AG, Havelaar AH, Friesema IH, et al. Risk factors for campylobacteriosis of chicken, ruminant, and environmental origin: a combined case-control and source attribution analysis. PLoS ONE. 2012;7(8):e42599. https://doi.org/10.1371/ journal.pone.0042599.

6. Di-Giannatale E, Garofolo G, Alessiani A, Di-Donato G, Candeloro L, Vencia W, et al. Tracing Back Clinical Campylobacter jejuni in the Northwest of Italy and Assessing Their Potential Source. Frontiers in Microbiology. 2016;13(7):887. https://doi.org/10.3389/fmicb.2016.00887.

7. Iovine NM. Resistance mechanisms in Campylobacter jejuni. Virulence. 2013:4:230-40.

8. Gharbi M, Bejaoui A, Hamda CB, Jouini A, Ghedira K, Zrelli C, Hamrouni $\mathrm{S}$, et al. Prevalence and antibiotic resistance patterns of Campylobacter spp. isolated from broiler chickens in the North of Tunisia. Biomed Res Int. 2018. https://doi.org/10.1155/2018/7943786.

9. Bankole HS, Baba-Moussa F, Agbankpe JA, Dougnon TV, Legonou M, Toukourou F, Baba-Moussa L. Essai d'isolement de Campylobacter dans la viande de volaille en République du Bénin. Int J Biol Chem Sci. 2012;6(5):1979-86.

10. Food and Agriculture Organization (FAO). Secteur Avicole Bénin. Revues nationales de l'élevage de la FAO, Production et Santé Animales. 2015; No. 10. Rome, $\mathrm{pp}: 74$

11. Kougblenou SD, Agbankpe AJ, Behanzin JG, Dougnon TV, Aniambossou A, Baba-Moussa L, Bankole HS. Microbiological safety and sanitary quality of primary production of leafy vegetables at Houeyiho and Seme-Kpodji vegetables farms in Southern Benin: risk factors for Campylobacter spp. Int J Food Sci. 2019. https://doi.org/10.1155/2019/8942608.

12. Dabboussi F, Alam S, Mallat H, Hlais S, Hamze M. Preliminary study on the prevalence of Campylobacter in childhood diarrhoea in north Lebanon. Eastern Mediterranean Health J. 2012;18(12):1225-8.

13. Linton D, Owen RJ, Stanley J. Rapid identification by PCR of the genus Campylobacter and of five species enteropathogenic for man and animals. Res Microbiol. 1996;147:707-18.

14. Talhouk RS, El-Dana RA, Araj GF, Barbour E, Hashwa F. Prevalence, antimicrobial susceptibility and molecular characterization of Campylobacter isolates recovered from humans and poultry in Lebanon. J Med Libanais. 1998;46(6):310-6.

15. The European Committee on Antimicrobial Susceptibility Testing (EUCAST). Breakpoint tables for interpretation of MICs and zone diameters. 2018; Version.2.0, http://www.eucast.org.

16. Thakur S, Zhao S, McDermott FP, Harbottle H, Abbott J, English L, Gebreyes WA, White DG. Antimicrobial resistance, virulence, and genotypic profile comparison of Campylobacter jejuni and Campylobacter coli isolated from humans and retail meats. Foodborne Pathogens Dis. 2010;7(7):835-44.

17. Szczepanska B, Andrzejewska M, Spica D, Klawe JJ. Prevalence and antimicrobial resistance of Campylobacter jejuni and Campylobacter coli isolated from children and environmental sources in urban and suburban areas. BMC Microbiol. 2017;17(1):80.

18. Asakura H, Sakata J, Nakamura H, Yamamoto S, Murakami S. Phylogenetic diversity and antimicrobial resistance of campylobacter coli from humans and animals in Japan. Microbes Environ. 2019;34(2):146-54. https://doi. org/10.1264/jsme2.ME18115.

19. Codex Alimentarius Commission (CAC). Code of hygienic practice for meat. Adopted by the CAC, New Zealand, July 2005 (CAC/RCP58*2005). Geneva, Switzerland: Codex Alimentarius Commission.

20. Kim HJ, Kim JH, Kim YI, Choi JS, Park MY, Nam H-M, Jung S-C, Kwon JW, Lee CH, Kim YH, Ku BK, Lee YJ. Prevalence and Characterization of Campylobacter spp isolated from domestic and imported poultry meat in Korea, 2004-2008. Foodborne Pathogens Dis. 2010;7(10):1203-9. https://doi. org/10.1089/fpd.20.

21. Varga C, Guerin MT, Brash ML, Slavic D, Boerlin P, Susta L. Antimicrobial resistance in Campylobacter jejuni and Campylobacter coli isolated from small poultry flocks in Ontario, Canada: a two-year surveillance study. PLoS ONE. 2019;14(8):e0221429. https://doi.org/10.1371/journ al.pone. 0221429 .

22. Adiguzel MC, Sigirci BD, Celik B, Kahraman BB, Metiner K, Ikiz S, Bagcigil AF, Ak S, Ozgur NY. Phenotypic and genotypic examination of antimicrobial resistance in thermophilic Campylobacter species isolated from poultry in Turkey. J Vet Res. 2018;62(4):463-8. https://doi.org/10.2478/ jvetres-2018-0071.

23. Lim S-K, Moon D-C, Chae MH, Kim HJ, Nam H-M, Kim S-R, Jang G-C, Lee K, Jung S-C, Lee HS. Macrolide resistance mechanisms and virulence factors in erythromycin-resistant Campylobacter species isolated from chicken and swine feces and carcasses. J Vet Med Sci. 2016;78(12):1791-5. https:// doi.org/10.1292/jvms.16-0307.

24. Bouhamed R, Bouayad L, Messad S, Zenia S, Naïm M, Hamdi TM. Sources of contamination, prevalence, and antimicrobial resistance of thermophilic Campylobacter isolated from turkeys. Vet World. 2018;11(8):1074-81.

25. Khan AJ, Rathore RS, Abulreesh HH, Qais FA, Ahmad I. Prevalence and antibiotic resistance profiles of Campylobacter jejuni isolated from poultry meat and related samples at retail shops in Northern India. Foodborne Pathogens Dis. 2018;15(4):218-25. https://doi.org/10.1089/fpd.2017.2344.

26. Elhadidy M, Miller WG, Arguello H, Alvarez-Ordoñez A, Duarte A, Dierick $\mathrm{K}$, et al. Genetic basis and clonal population structure of antibiotic resistance in Campylobacter jejuni isolated from broiler carcasses in Belgium. Front Microbiol. 2018;17(9):1014. https://doi.org/10.3389/fmicb 2018.01014

27. Giacomelli M, Salata C, Martini M, Montesissa C, Piccirillo A. Antimicrobial resistance of Campylobacter jejuni and Campylobacter coli from poultry in Italy. Microbial Drug Res. 2014;20(2):181-8.

28. Garcia-Migura L, Hendriksen RS, Fraile L, Aarestrup FM. Antimicrobial resistance of zoonotic and commensal bacteria in Europe: the missing link between consumption and resistance in veterinary medicine. Vet Microbiol. 2014;170(2):1-9. https://doi.org/10.1016/j.vetmic.2014.01.013.

29. Chukwu MO, Abia ALK, Ubomba-Jaswa E, Obi L, Dewar JB. Characterization and phylogenetic analysis of campylobacter species isolated from paediatric stool and water samples in the Northwest Province, South Africa. Int J Environ Res Public Health. 2019;16:2205. https://doi. org/10.3390/ijerph16122205.

\section{Publisher's Note}

Springer Nature remains neutral with regard to jurisdictional claims in published maps and institutional affiliations. 